\title{
The Development and Validation of a Measure of Health-Related Quality of Life for Non-Hodgkin's Lymphoma: The Functional Assessment of Cancer Therapy_Lymphoma (FACT-Lym)
}

\author{
Fay J. Hlubocky, ${ }^{1,2}$ Kimberly Webster, ${ }^{1}$ John Cashy, ${ }^{1}$ Jennifer Beaumont, ${ }^{1}$ and David Cella ${ }^{1}$ \\ ${ }^{1}$ Department of Medical Social Sciences, Robert H. Lurie Comprehensive Cancer Center and Feinberg School of Medicine, \\ Northwestern University, Chicago, IL 60611, USA \\ ${ }^{2}$ Section of Hematology and Oncology, Department of Medicine, MacLean Center for Clinical Medical Ethics, The University of Chicago, \\ Chicago, IL 60637, USA
}

Correspondence should be addressed to Fay J. Hlubocky; fhlubock@medicine.bsd.uchicago.edu

Received 30 July 2012; Accepted 21 December 2012

Academic Editor: Vincent Ribrag

Copyright (C) 2013 Fay J. Hlubocky et al. This is an open access article distributed under the Creative Commons Attribution License, which permits unrestricted use, distribution, and reproduction in any medium, provided the original work is properly cited.

Background. The individual concerns of non-Hodgkin's lymphoma (NHL) patients require identification and assessment during clinical research proposing to measure patients' outcomes. The FACT-Lym was developed as part of the FACIT measurement system to address health-related quality-of-life (HRQL) issues for NHL patients. Patients and Methods. Items for the FACT lymphoma subscale (LymS) were generated from healthcare provider interviews, published literature, and content validity patient interviews. The FACT-Lym was validated on a sample of 84 NHL patients, with additional measures at baseline (T1), 3-7 days (T2), and 8-12 weeks (T3). Results. Item correlations, expert relevance ratings, and patient input on content shortened the initial 22 -item LymS to 15 items. The validation sample included $56 \%$ female, $76.2 \%$ white, $60 \%$ indolent disease, and $85 \%$ receiving treatment. Internal consistency coefficients for the 15 -item $\operatorname{LymS}(.79, .85$, and .84 T1-T3) and test-retest stability (.84) indicated good reliability. Correlations between LymS and SF-36 physical $(r=.62)$ and mental $(r=.48)$ summary scores reflect concurrent validity. Responsiveness to ECOG performance status and treatment status exceeded established FACT subscale scores. The FACTLymS differentiated patients' retrospective ratings of change in each of the three groups (better; unchanged; worse), $P<0.001$. Conclusions. These results support the validity of the FACT-Lym and suggest it will be a useful targeted endpoint in NHL clinical research.

\section{Introduction}

Non-Hodgkin's Lymphoma (NHL) affects approximately 66,000 Americans every year and is the sixth most common cancer among Americans. Five-year survival is better than $60 \%$ and 10 -year relative survival is over $50 \%$ [1]. Significant advances in the clinical management of indolent and aggressive NHL-utilizing cancer therapeutics have improved both response and survival rates for patients thus making it a chronic disease [2,3]. Autologous and allogeneic stem cell transplantation have shown to improve patient's outcomes including long-term survival [4-8]. Several treatment options promote disease remission or stability and can minimize side effects such as anemia, mucositis, and myelosuppression [9-12]. Psychological morbidity can also be reduced or managed [13-17]. However, despite improvement in survival and patient management, patients must still endure treatment toxicities with uncertain benefit to any individual treated. The demands of comorbid conditions, psychological distress, and general disease burden must be endured and balanced against treatment efficacy $[8,9,16-$ 22]. Recent work has examined the longitudinal effects of 
disease and treatment, including quality-of-life considerations and the cost-effectiveness of treatment [22-33]. NHL treatment has often been evaluated without a specific focus on the self-reported concerns and symptoms of patients. There is currently no validated NHL-specific health-related quality-of-life (HRQL) instrument available for use in clinical research. We report on the development and validation of a patient-reported HRQL outcome questionnaire that is specifically relevant to NHL $[34,35]$. The primary purpose of the instrument is to provide an outcome instrument for clinical research. This lymphoma subscale (LymS) is designed to complement the 27-item Functional Assessment of Cancer Therapy-General (FACT-G) questionnaire which is part of the larger Functional Assessment of Chronic Illness Therapy Measurement System [36-40].

\section{Materials and Methods}

2.1. Scale Development. The development of the FACTlymphoma subscale (LymS) occurred in three phases: item generation, item reduction, and scale construction [36-40]. The item-generation phase to identify content relevant to NHL was obtained through literature review and interviews with health care providers. Supplemental content validity interviews were conducted with patients during the validation phase. These data were used as the foundation from which to build the NHL-specific subscale. While the disease trajectories and management of patients with leukemia and lymphoma have important distinctions, many HRQL concerns are common across hematological malignancies $[16,19,41]$. Therefore, the development of the LymS was initiated with the FACT-Leukemia subscale as a foundation to modify based on literature, expert, and patient input. Patient feedback determined appropriateness of the content included.

Of the fifteen providers invited to participate, 12 accepted, 1 declined, and 2 did not respond. Of the twelve acceptances, 8 (7 physicians and 1 nurse) participated providing complete data. These 8 expert providers ( 7 from USA and 1 from UK) were interviewed by one of the authors (DC) or completed interview materials by self-administration. Experts in the areas of medical oncology, hematology/oncology, and bone marrow/stem cell transplantation had been practicing for at least 10 years (range $10-26$ years) and treated or advised 750 6,000 lymphoma patients during years of practice. Providers contributed their educated opinions regarding how lymphoma affects a patient's physical well-being, functional wellbeing, emotional well-being, and other disease or treatmentrelated issues (e.g., cognition). Providers assigned ratings for the identified symptoms on a scale of 0 ("not at all common") to 3 ("extremely common"). A second rating evaluated how important a symptom/issue is to NHL patients as rated on a scale of 0 ("not at all important") to 3 ("extremely important"). Experts completed an item-rating survey of 53 symptoms and issues based on the literature and our prior research in hematologic malignancies and were asked to rate how common and important each of these symptoms is to NHL patients on a scale of 0 ("not at all common") to 3 ("extremely common").

From these interviews, 118 symptoms/concerns were identified: 27 disease-based physical symptoms (e.g., pain; fever), 5 functional (e.g., insomnia; itching from Sézary's syndrome), 17 emotional (e.g., depression; anxiety), 5 family (e.g., support issues; family communication issues), 7 sexual/intimacy (e.g., inability to have sex; loss of interest), 8 social (e.g., social isolation), 4 work (e.g., impaired ability to work because of risks or disease), 10 cognitive (e.g., cognition; concern over impairment), 5 future orientation (e.g., fear; uncertainty of relapse), 6 other, and 24 treatment related. When combined with the literature review summary, frequency referencing determined relevance and weight (degree of representation) in subsequent phases of item reduction.

In the item-reduction phase, redundant, overlapping, and irrelevant items were eliminated reducing the item pool to 69 items (52 disease related; 17 treatment related). These symptoms included 18 disease-related physical symptoms, 3 functional, 14 emotional, 3 family, 4 sexual/intimacy, 3 social, 4 occupational, 1 cognitive, 2 future orientation, and 17 treatment related. The next phase of item reduction involved evaluation of the 69 remaining items by a second group of 16 expert health care providers (providers included 7 from the original item-generation sample and 9 new participants: 15 physicians and 1 nurse). The 69 symptoms/issues were formatted according to the same item-rating survey used to evaluate the initial set of 53 items and administered to providers. During the scale construction phase, data were evaluated and set additional criteria for item retention. At this stage, item content (overlap with exiting FACT$G$ items or other items within group), degree of disease specificity, redundancy, and clinical relevance were used to guide decision making. A near-final list of 22 items for the FACT-Lymphoma was reconciled with the FACIT item database; 19 of 22 existing items were recruited, and 3 new items were written and reviewed by our multilingual translation team to ensure items were written in a manner that would lend well to translations. A final set of 22 items for the LymS was constructed to complement the existing FACT-G questionnaire, similar in form to other diseasespecific scales of the FACIT measurement system (see the appendix in the Supplementary Material available online at http://dx.doi.org/10.1155/2013/147176). The response choices are identical to the five-point Likert scale of the FACT-G $(0=$ Not at all; 1 = a little bit; 2 = somewhat; 3 = quite a bit; $4=$ very much).

2.2. Scale Validation Sample. In order to test the initial psychometric properties of the LymS, 84 NHL patients were recruited from three different urban Midwestern academic medical centers in one city. Potential participants were identified by chart review and physician referral. Eligibility criteria included (1) at least 2 months after diagnosis of NHL; (2) able to understand and provide informed consent; (3) provision of written informed consent; (4) fluency in English; (5) at least 18 years of age; (6) life expectancy of $>3$ months; (7) no current diagnosis of psychosis or dementia. 
TABLE 1: Demographic characteristics of the sample.

\begin{tabular}{|c|c|}
\hline Demographic characteristics & $\begin{array}{c}\text { Patient sample } \\
(N=84) \\
N(\%)\end{array}$ \\
\hline \multicolumn{2}{|l|}{ Age, years } \\
\hline Median & 62 \\
\hline Range & $23-82$ \\
\hline \multicolumn{2}{|l|}{ Gender } \\
\hline Male & $37(44.0 \%)$ \\
\hline Female & $47(56.0 \%)$ \\
\hline \multicolumn{2}{|l|}{ Race/ethnicity } \\
\hline White & $64(76.2 \%)$ \\
\hline White, other & $8(9.5 \%)$ \\
\hline White, Amer. Indian/Alaskan Native & $1(1.2 \%)$ \\
\hline Black & $3(3.6 \%)$ \\
\hline Hispanic (Mexican), other & $2(2.4 \%)$ \\
\hline Asian & $2(2.4 \%)$ \\
\hline Other & $1(1.2 \%)$ \\
\hline Missing & $3(3.6 \%)$ \\
\hline \multicolumn{2}{|l|}{ Marital status } \\
\hline Never married & $7(8.3 \%)$ \\
\hline Married & $58(69.0 \%)$ \\
\hline Living with partner & $2(2.4 \%)$ \\
\hline Divorced & $7(8.3 \%)$ \\
\hline Widowed & $10(11.9 \%)$ \\
\hline \multicolumn{2}{|l|}{ Years of education } \\
\hline 6 th grade & $1(1.2 \%)$ \\
\hline 8th grade & $2(2.4 \%)$ \\
\hline 11th grade & $1(1.2 \%)$ \\
\hline High school grad/GED & $14(16.7 \%)$ \\
\hline Some college & $18(21.4 \%)$ \\
\hline College degree (B.A./B.S.) & $30(35.7 \%)$ \\
\hline Advanced degree (M.S., Ph.D., M.D.) & $17(20.2 \%)$ \\
\hline Missing & $1(1.2 \%)$ \\
\hline
\end{tabular}

2.3. Assessments. The FACT-Lym was validated on this sample of 84 NHL patients, who completed it with other measures at baseline (T1), 3-7 days (T2), and 8-12 weeks (T3). Performance status rating (PSR), sociodemographics, clinical information, and treatment history were collected on all patients at baseline. The FACT-Lym, consisting of the 27-item FACT-G and the new 22-item developed LymS, was administered to all patients at all three timepoints [37-40]. The FACT-G assesses four dimensions of HRQL: physical well-being (PWB, 7 items), social/family well-being (SWB, 7 items), emotional well-being (EWB, 6 items), and functional well-being (FWB, 7 items).

In addition to the FACT-Lym, a battery of previously validated self-report measures was administered to evaluate reliability, convergent, and divergent validity. Additional selfreport measures included Eastern Cooperative Oncology
Group Performance Status Rating (ECOG PSR) [42]; Profile of Mood States-Short Form (Brief POMS) at T1 and T3 [43]; Marlowe-Crowne Social Desirability Scale-Short Form at T1 [44]; Medical Outcomes Study Short-Form Health Survey Version 1 (SF-36) [45] at T1 and T3; and the Global Rating of Change Scale [46] at T3 only. The ECOG PSR is a widely used functional status rating (range 0-4) for cancer patients. The Brief POMS is an 11-item measure of recent affective state, which yields a total mood disturbance score and subscale scores for depression, tension, confusion, and fatigue. The Marlowe-Crowne Social Desirability Scale is a self-report measure of social desirability. The SF-36 is a self-report instrument used to assess general HRQL and yields two overall indices, the Physical Component Summary (PCS) and the Mental Component Summary (MCS). Patients completed an open-ended content validity interview to elicit feedback on the appropriateness of existing FACT-Lym items and the possible absence of other relevant lymphoma-related concerns.

After informed consent, patients were encouraged to complete the forms during their clinic visit/hospital stay but were allowed to return completed forms in the mail or by telephone interview within a week. Patients were compensated a total of $\$ 60.00$ for completion of all 3 assessments.

2.4. Statistical Considerations. The 22 -item LymS was scored in the same manner as the FACT-G: summing of the 0-4 responses, with reverse coding of items as needed so that a high score reflects good HRQL (http://www.facit.org/). Adding the 22-item FACT-LymS score (possible range = $0-88$ ) to the FACT-G total score (possible range $=0$ 108) provides a total FACT-Lym score (possible range $=0$ 196). Five subscale scores and an overall HRQL score can be calculated, with higher scores reflecting better HRQL. A summary index of physical/functional outcomes is also obtained using the Trial Outcome Index (TOI: sum of the physical, functional, and lymphoma-specific concerns). Internal consistency (alpha $(\alpha)$ ) coefficients were computed for all subscales to reflect the interrelatedness of a set of aggregated questions [47]. A coefficient $\geq 0.70$ is generally regarded as acceptably high for aggregation of responses into a single score [48]. The 22-item LymS was reviewed for item redundancy in order to create a shortened, yet reliable and valid, scale for subsequent analyses. Convergent validity of the FACT-Lym was tested by examining the Spearman correlation coefficients among the FACT-Lym (subscales and total scores) and related measures. To test criterion-related validity, score differences were evaluated according to differences in clinical characteristics (e.g., PSR and hemoglobin). An analysis of variance was performed on subscale and total scores to examine the expected relationship between performance status and HRQL. Patients were divided into three performance status groups: 0 , normal functioning without symptoms; 1 , some symptoms but no extra rest required; and 2-4, each reflecting varying degrees of bed rest needed during the waking day. SAS version 8.02 was used for the statistical analyses (SAS Institute, Cary, NC, USA). 
TABLE 2: Clinical characteristics of the sample at baseline.

Clinical characteristics

Patient sample

$(N=84)$

$N(\%)$

NHL type

B Cell

$77(91.6 \%)$

T Cell

$5(6.0 \%)$

Other

$1(1.2 \%)$

Missing

$1(1.2 \%)$

NHL grade

Low

$50(59.5 \%)$

Intermediate

$22(26.2 \%)$

High

$12(14.3 \%)$

Extent

Single site (excluding abdomen/mediastinum)

$6(7.1 \%)$

Disease above the diaphragm (including mediastinum)

15 (17.9\%)

Disease below the diaphragm (including GI disease)

$21(25.0 \%)$

Disease on both sides of the diaphragm

$19(22.6 \%)$

Any of the above with bone marrow or CNS involvement

$22(26.2 \%)$

Missing

$1(1.2 \%)$

Current active treatment?

Yes

$71(84.5 \%)$

No

$13(15.5 \%)$

Platelets, $1000 / \mu \mathrm{L}$ ( $n=1$ missing)

Mean

Range

16-834

SD

116.2

White blood cells ( $n=1$ missing)

Mean

Range

SD

Red blood cells ( $n=1$ missing)

Mean

Range

SD

Hemoglobin, g/dL ( $n=1$ missing)

Mean

Range

7-16

SD

Patient-rated ECOG PSR

0 , normal activity

1 , some symptoms, no rest

2 , bed rest $<50 \%$ of day 
TABLE 2: Continued.

\begin{tabular}{lc}
\hline Clinical characteristics & $\begin{array}{c}\text { Patient sample } \\
(N=84) \\
N(\%)\end{array}$ \\
\hline Physician-rated ECOG PSR & $42(50.0 \%)$ \\
0, normal activity & $36(42.8 \%)$ \\
1, some symptoms, no rest & $4(4.8 \%)$ \\
2, bed rest $<50 \%$ of day & $2(2.4 \%)$ \\
Missing & \\
\hline
\end{tabular}

TABLE 3: Descriptive statistics at the baseline assessment for the FACT-Lym scales.

\begin{tabular}{|c|c|c|c|c|c|c|c|}
\hline \multirow[b]{2}{*}{$\begin{array}{l}\text { FACT scale or subscale } \\
\text { (number of items) }\end{array}$} & \multicolumn{3}{|c|}{ Means (SD) } & \multicolumn{3}{|c|}{ Cronbach's alpha } & \multirow{2}{*}{ ICC } \\
\hline & Time $1(n=84)$ & Time $2(n=74)$ & Time $3(n=71)$ & Time $1(n=84)$ & Time $2(n=74)$ & $\begin{array}{c}\text { Time } 3 \\
(n=71)\end{array}$ & \\
\hline Physical (7) & $23.3(4.2)$ & $21.9(5.0)$ & $22.4(5.0)$ & 0.82 & 0.82 & 0.85 & .65 \\
\hline Social/family (7) & $23.5(4.7)$ & $22.9(4.7)$ & $23.5(4.7)$ & 0.84 & 0.78 & 0.84 & .61 \\
\hline Emotional (6) & $18.8(3.8)$ & $19.5(3.5)$ & $19.3(3.6)$ & 0.70 & 0.74 & 0.80 & .77 \\
\hline Functional (7) & $20.3(5.7)$ & $19.3(6.0)$ & $19.3(5.8)$ & 0.87 & 0.89 & 0.89 & .85 \\
\hline Lymphoma (15) & $47.6(7.8)$ & $47.9(8.8)$ & $48.6(7.8)$ & 0.79 & 0.85 & 0.84 & .84 \\
\hline TOI (29) & $91.2(15.4)$ & $89.0(17.9)$ & $90.2(17.3)$ & 0.91 & 0.93 & 0.94 & .87 \\
\hline FACT-G (27) & $86.0(13.5)$ & $83.5(14.6)$ & $84.5(15.2)$ & 0.87 & 0.90 & 0.94 & .84 \\
\hline FACT-Lym (42) & $133.6(19.9)$ & $131.4(22.4)$ & $133.1(22.0)$ & 0.90 & 0.93 & 0.95 & .87 \\
\hline
\end{tabular}

\section{Results}

3.1. Demographic and Clinical Characteristics. A total of 129 patients were approached for participation in the validation study. Of these, 32 (25.0\%) declined study participation and 12 $(9.3 \%)$ were ineligible. One patient provided written consent but failed to provide study data. Reasons for refusal included too ill $(n=2)$; too tired $(n=1)$; time-consuming study $(n=7)$; not interested $(n=16)$; nurse refusal $(n=2)$; lack of time $(n=1)$; and too upset $(n=1)$. A total of 84 subjects completed T1 assessment, 74 (88.1\%) completed T2 assessment, and $71(84.5 \%)$ patients completed T3 assessment. Missed assessments were due to death $(n=1)$; illness $(n=2)$; loss to followup $(n=4)$; and passive refusal $(n=6)$.

The demographic and clinical characteristics of the sample are summarized in Tables 1 and 2. Patients were mostly female (56\%), white (76.2\%), and married (69.0\%) with $95.2 \%>$ high school education. They had primarily B-Cell histology (91.7\%) and low-grade disease (59.5\%). Extent of disease was mixed and included patients in all categories. Most patients (84.5\%) were receiving some form of treatment at baseline. PSR was generally high with over half of the sample (53.6\%) asymptomatic and functioning well.

3.2. Content Validity. Baseline NHL patients completed a content validity questionnaire following $\mathrm{T} 1$ assessment to inform item reduction. Patients evaluated items related to the LymS and the majority of patients-reported items were relevant, comprehensive, and self-explanatory. Some patients stated that items were relevant to the time of active treatment or time of diagnosis. For example, 5 patients reported they had no experience with B symptom items; 4 denied any pain issues; 1 could not recognize night sweats as a symptom; 2 did not understand the item "discomfort or pain in the stomach area." A few patients stated uncertainty about future health was not specific to lymphoma.

Based upon patient's feedback, despite evidence that the 22-item FACT-LymS demonstrated satisfactory psychometric properties, we completed a second item reduction process to minimize patient burden and maximize efficiency. Validation data on individual items were evaluated for conceptual overlap, specificity, relevance, and comprehension. Team review using item correlations on the 22 -item subscale, expert relevancy ratings from the development phase of the study, and patient input on content shorten the subscale from 22 to 15 items. Reasons for item deletion included overlap with other items (redundancy); low relevance ratings; low prevalence, and gender bias. The appendix lists the 15 retained items and the 7 items recommended for removal.

3.3. Descriptive Statistics. Table 3 presents descriptive statistics for the FACT-Lym subscales, total score, and Trial Outcome Index (TOI) at each assessment for the pooled patient sample. All subscales and aggregated scores showed high internal consistency at initial assessment (Cronbach's alpha $(\alpha)$ range, .70-.91) and retesting at T2 (Cronbach's alpha $(\alpha)$ range, .74-.93) and T3 (Cronbach's alpha ( $\alpha$ ) range, .80 to .95). The 15-item LymS demonstrated good internal consistency $(\alpha$ $=.79, .85$, and .84 at Times 1,2 , and 3 resp.), supporting its use as a measure of disease-related symptoms and concerns. Intraclass correlation coefficients were calculated to evaluate test-retest reliability of the subscales. 
TABLE 4: Convergent and divergent validity of the FACT-Lym at baseline (Spearman's correlation coefficients).

\begin{tabular}{cccccccccc}
\hline & Variable & $N$ & PWB & SWB & EWB & FWB & LymS & Total & TOI \\
\hline 1 & POMS_T & 83 & -0.13 & $-0.34^{\dagger}$ & $-0.59^{\ddagger}$ & $-0.28^{*}$ & $-0.33^{\dagger}$ & $-0.43^{\ddagger}$ & $-0.33^{\dagger}$ \\
2 & POMS_D & 83 & $-0.39^{\ddagger}$ & $-0.43^{\ddagger}$ & $-0.59^{\ddagger}$ & $-0.47^{\ddagger}$ & $-0.54^{\ddagger}$ & $-0.62^{\ddagger}$ & $-0.54^{\ddagger}$ \\
3 & POMS_F & 82 & $-0.50^{\ddagger}$ & $-0.51^{\ddagger}$ & $-0.46^{\ddagger}$ & $-0.53^{\ddagger}$ & $-0.62^{\ddagger}$ & $-0.67^{\ddagger}$ & $-0.64^{\ddagger}$ \\
4 & POMS_C & 81 & $-0.27^{*}$ & -0.20 & $-0.40^{\ddagger}$ & $-0.23^{*}$ & $-0.44^{\ddagger}$ & $-0.42^{\ddagger}$ & $-0.39^{\ddagger}$ \\
5 & POMS_TMD & 83 & $-0.42^{\ddagger}$ & $-0.47^{\ddagger}$ & $-0.65^{\ddagger}$ & $-0.50^{\ddagger}$ & $-0.60^{\ddagger}$ & $-0.68^{\ddagger}$ & $-0.60^{\ddagger}$ \\
6 & MCS & 82 & $0.40^{\ddagger}$ & $0.29^{\dagger}$ & $0.52^{\ddagger}$ & $0.46^{\ddagger}$ & $0.48^{\ddagger}$ & $0.58^{\ddagger}$ & $0.53^{\ddagger}$ \\
7 & PCS & 82 & $0.64^{\ddagger}$ & 0.15 & 0.15 & $0.56^{\ddagger}$ & $0.62^{\ddagger}$ & $0.57^{\ddagger}$ & $0.68^{\ddagger}$ \\
8 & MC-10 & 83 & -0.06 & 0.16 & -0.02 & 0.03 & -0.15 & -0.04 & -0.10 \\
\hline
\end{tabular}

${ }^{*} P<0.05$.

${ }^{\dagger} P<0.01$.

${ }^{\ddagger} P<0.001$.

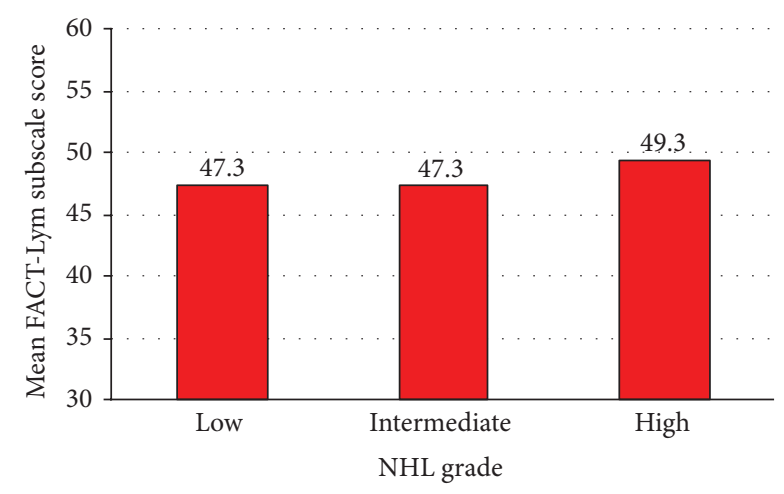

Figure 1: FACT-Lym subscale score by NHL grade.

3.4. Known Group Comparisons. FACT-Lym scores did not differentiate between patient groups defined by NHL grade (ANOVA $P=0.708$ ). Figure 1 depicts mean LymS scores at baseline for each group. Effect sizes (mean difference/standard deviation) for the differences between adjacent groups were 0.01 (low versus intermediate) and 0.26 (intermediate versus high). All FACT-Lym subscales and aggregated scores showed differences based upon PSR, with the highest mean score associated with the best PSR $(P<0.001$, Figure 2). The incremental difference was approximately 68 points from one PSR category to the next. This difference, in excess of 0.5 standard deviation per adjacent category comparison, exceeds the threshold for a meaningful score difference. Figure 3 depicts NHL patients currently receiving some form of therapy (radiation, chemotherapy, etc.) compared to those receiving no therapy. Patients currently receiving therapy demonstrated lower FACT-LymS scores (poorer HRQL; $P=0.002$ ). This difference is approximately 7 points, nearly a full standard deviation unit.

3.5. Convergent/Divergent Validity. Spearman's $\rho$ correlation coefficients assessed the strength of relationships across FACT measures (FACT-G subscales; LymS; TOI; see Table 4). Correlations with the POMS total score were moderately high (0.42 to 0.68), as were correlations with the SF-36 MCS ( $r=$ $0.48, P<0.001)$ and PCS $(r=0.62, P<0.001)$. Regarding

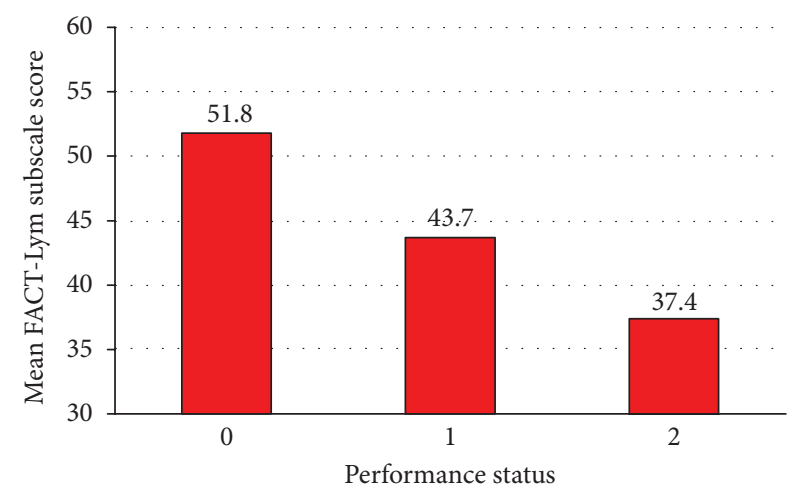

FIGURE 2: FACT-Lym subscale score by self-reported performance status.

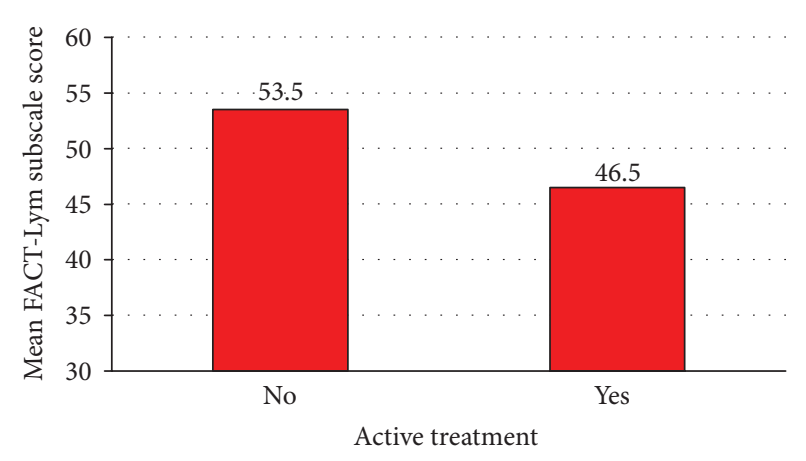

FIGURE 3: FACT-Lym subscale score by current treatment.

divergent validity, association between FACT subscales and social desirability (MC-10 score) was consistently near zero, with the highest coefficient (.16) between social desirability and social family well-being.

3.6. Sensitivity to Change. Figure 4 displays the FACT-Lym subscale change scores over time for three groups defined by patients' retrospective ratings of change at the final assessment. The FACT-LymS differentiated patients in each of the three groups (worse; unchanged; better), $P<0.001$ (Figure 4). Figure 5 displays the FACT-Lym Subscale change 


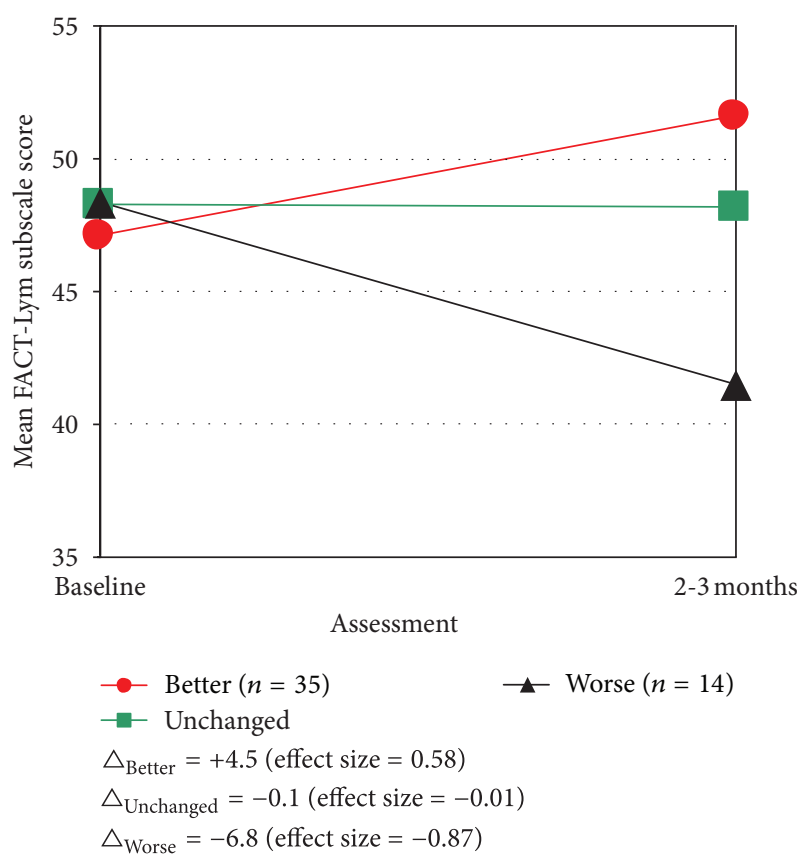

FIgure 4: Change in FACT-Lym Subscale Score by Global Rating of Change $(P<.001)$.

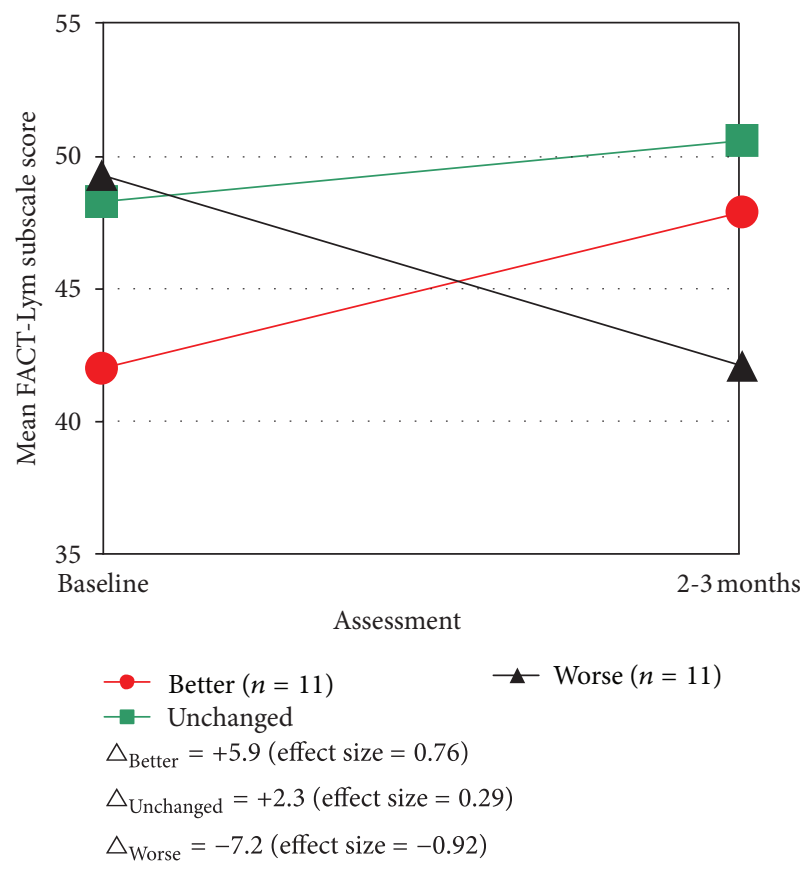

Figure 5: Change in FACT-Lym subscale score by change in performance status $(P<.001)$.

scores over time for three groups defined by change over 3 months in patient's performance status. The FACT-LymS differentiated patients in each of the three groups (better; unchanged; worse), $P<0.001$ (Figure 5). Sufficiently large effect sizes (e.g., over 0.50 ) were observed.
3.7. Minimally Important Difference. Distribution-based estimates of a likely MID have typically fallen in the 0.33 to $0.50 \mathrm{SD}$ range. For the LymS, this corresponds to 2.6 to 3.9 points. The standard error of measurement (SD multiplied by the square root of one minus the test-retest reliability) is 3.0. Anchor-based group differences and change scores presented in the preceding tables and figures ranged from 4.5 to 8.1. Several of these differences are equivalent to an effect size far exceeding 0.50 and likely represent an overestimate of the minimally important difference. Nine patients reported that their lymphoma-related symptoms were "a little worse" at $\mathrm{T} 3$ relative to baseline. These patients had a mean decrease in LymS of $5.1(S D=9.1)$. The 11 patients who reported symptoms were "a little better" had a mean increase of 4.2 (SD $=7.6$ ). This evidence suggests that a likely MID range for the LymS is approximately $3-5$ points or $5-8 \%$ of the scale range $(0-60)$.

\section{Discussion}

Advances in the clinical management of non-Hodgkin's lymphoma (NHL) have led to the availability of less toxic therapies for patients. However, the specific self-reported HRQL concerns of NHL patients undergoing such cancer treatment, including clinical trials, remain unknown. To date, no available site-specific instrument assesses the targeted issues and symptoms associated with NHL. The FACT-Lym provides for a reliable and valid assessment of these unique issues, supplements the FACT-G, and is responsive to change in health status. The significance of the FACT-Lym in clinical research will require demonstration in subsequent longitudinal studies; however, data suggest the likely performance of such a tool in that setting.

The FACT-Lym was developed utilizing comprehensive input from NHL patients who rated item content derived from expert interviews and literature review. The 22 -item version of the LymS was reduced to a 15 -item version with comparable content coverage and equivalent performance across all analyses. Item content of the FACT-LymS (15- and 22-item versions) appears to be clinically relevant and comprehensive in coverage (content validity). The subscale demonstrated consistently high reliability. It also differentiated patients with differing NHL grade, performance status, and whether or not patients were in active therapy. The responsiveness of the LymS to change in self-reported symptoms and wellbeing exceeded criteria for meaningful differences. This suggests that the LymS will be a useful targeted endpoint in trials where lymphoma-specific concerns are paramount and that the 15-item version of the subscale, shortened based upon patient input, performs as well as the longer, 22-item version.

Several other considerations pertaining to the results presented here deserve further attention. For instance, the correlations between FACT-LymS and other FACT scales with the POMS total score (range $=-0.42$ to 0.68 ) and with the SF-36 MCS $(r=0.48, P<0.001)$ and PCS $(r=0.62$, $P<0.001)$ were moderately strong. This implies that FACTLym drums into both the physical and mental aspects of 
self-reported health almost equally. A thorough item review of the content of the LymS questions reveals that the issues patients and experts consider to be of significance relative to lymphoma are physical (pain, swelling, and fatigue) and psychological (worry, emotional) in nature.

Several study limitations should be noted. Most (85\%) patients were receiving chemotherapy; thus, generalizability to other patient groups is uncertain. Regarding attrition, 13 patients did not provide follow-up (T2 and T3) data after baseline evaluation due to death; illness; loss to followup; and passive refusal. Although there were no baseline differences in patient's characteristics or self-reported health status between the remaining 74 and the 10 who did not provide a second assessment, it remains possible that dropout was not random and may have influenced the results. We believe that the slightly reduced level of power achieved with our final sample does not greatly affect the interpretation of our results. While the loss of 6 patients due to passive refusal may constitute nonrandom dropout, it comprises less than $10 \%$ of the total sample and is unlikely to have a major impact on conclusions regarding scale reliability and validity. The goal of demonstrating observed differences between clinically distinct groups is still achieved through the available data. Finally, the validity of the FACT-Lym for all lymphoma patients remains to be established. This validation study was conducted with a mixed group of lymphoma patients, most of whom had low-grade disease and were receiving rituximab-containing therapy (e.g., rituximab alone or RCHOP). Symptom experiences or priorities may vary by type of lymphoma (e.g., Burkitt versus follicular), yet the sample size was too small to evaluate this. Study of the FACT-Lym in homogeneous subgroups of patients (e.g., those enrolled into clinical trials) would further inform its utility and validity.

In summary, the FACT-Lym appears to be a valid and reliable measure for assessing the impact of lymphoma on HRQL. Pilot work has also identified that the FACT-Lym has shown good reliability and linguistic validity with three language versions [49]. The concerns and symptoms of NHL patients were sufficiently captured by self-report as tested by the content validity interview and attending validation analyses. The measure should prove useful to clinicians and researchers in clinical trial evaluations and possibly in guiding treatment decision making.

\section{Acknowledgment}

This work was supported by an investigator-initiated research grant from Eli Lilly and Company.

\section{References}

[1] R. Siegel, E. Ward, O. Brawley, and A. Jemal, "Cancer statistics, 2011," A Cancer Journal for Clinicians, vol. 61, no. 4, pp. 212-236, 2011.

[2] B. A. Bilodeau and K. L. Fessele, "Non-Hodgkin's lymphoma," Seminars in Oncology Nursing, vol. 14, no. 4, pp. 273-283, 1998.

[3] National Cancer Institute, Non-Hodgkins lymphoma, 2011, http://www.cancer.gov/cancertopics/types/non-hodgkin.
[4] G. A. Hale and G. L. Phillips, "Allogeneic stem cell transplantation for the non-Hodgkin's lymphomas and Hodgkin's disease," Cancer Treatment Reviews, vol. 26, no. 6, pp. 411-427, 2000.

[5] A. Olivieri, D. Capelli, M. Montanari et al., "Very low toxicity and good quality of life in 48 elderly patients autotransplanted for hematological malignancies: a single center experience," Bone Marrow Transplantation, vol. 27, no. 11, pp. 1189-1195, 2001.

[6] E. Vellenga, M. van Agthoven, A. J. Croockewit et al., "Autologous peripheral blood stem cell transplantation in patients with relapsed lymphoma results in accelerated haematopoietic reconstitution, improved quality of life and cost reduction compared with bone marrow transplantation: the Hovon 22 study, British Journal of Haematology, vol. 114, no. 2, pp. 319326, 2001.

[7] M. E. McFadden, "Malignant lymphomas," in Cancer Nursing Principles and Practice, S. L. Groenwald, M. H. Frogge, M. Goodman, and C. H. Yarbro, Eds., pp. 1200-1229, Jones and Bartlett Publishers, Boston, Mass, USA, 3rd edition, 1993.

[8] M. van Agthoven, E. Vellenga, W. E. Fibbe, T. Kingma, and C. A. Uyl-de Groot, "Cost analysis and quality of life assessment comparing patients undergoing autologous peripheral blood stem cell transplantation or autologous bone marrow transplantation for refractory or relapsed non-Hodgkin's lymphoma or Hodgkin's disease: a prospective randomised trial," European Journal of Cancer, vol. 37, no. 14, pp. 1781-1789, 2001.

[9] S. M. Hubbard and D. L. Longo, "Treatment-related morbidity in patients with lymphoma," Current Opinion in Oncology, vol. 3, no. 5, pp. 852-862, 1991.

[10] M. S. Kaminski, T. L. Kauf, A. D. Zelenetz et al., “Treatment of transformed and refractory low-grade lymphoma with Bexxar therapy is associated with improvements in quality of life," in Proceedings of the Annual Conference of the American Society of Hematology (ASH '01), 2001, abstract no 1790.

[11] M. Duvic, T. M. Kuzel, E. A. Olsen et al., "Quality-of-life improvements in cutaneous T-cell lymphoma patients treated with Denileukin Diftitox (ONTAK)," Clinical Lymphoma, vol. 2, no. 4, pp. 222-228, 2002.

[12] A. Österborg, Y. Brandberg, V. Molostova et al., "Randomized, double-blind, placebo-controlled trial of recombinant human erythropoietin, epoetin beta, in hematologic malignancies," Journal of Clinical Oncology, vol. 20, no. 10, pp. 2486-2494, 2002.

[13] D. Razavi, N. Delvaux, A. Brédart et al., "Professional rehabilitation of lymphoma patients: a study of psychosocial factors associated with return to work," Supportive Care in Cancer, vol. 1, no. 5, pp. 276-278, 1993.

[14] F. R. Loberiza Jr., J. D. Rizzo et al., "Depressive syndrome and early deaths following stem cell transplantation for hematologic disease," in Proceedings of the Annual Conference of the American Society of Hematology (ASH '01), 2001, abstract no 3091.

[15] R. Pettengell, C. Donatti, P. Hoskin et al., "The impact of follicular lymphoma on health-related quality of life," Annals of Oncology, vol. 19, no. 3, pp. 570-576, 2008.

[16] K. Webster and D. Cella, "Quality of life in patients with lowgrade non-Hodgkin's lymphoma," Oncology, vol. 12, no. 5, pp. 697-714, 1998.

[17] P. Allart, P. Soubeyran, and F. Cousson-Gélie, “Are psychosocial factors associated with quality of life in patients with haematological cancer? A critical review of the literature," PsychoOncology, 2012.

[18] J. Fernsler and J. S. Fanuele, "Lymphomas: long-term sequelae and survivorship issues," Seminars in Oncology Nursing, vol. 14, no. 4, pp. 321-328, 1998. 
[19] L. Lesko, "Hematopoietic dyscrasias," in Psycho-Oncology, H. C. Holland, Ed., pp. 406-416, Oxford university Press, New York, NY, USA, 1998.

[20] S. K. Smith, S. Zimmerman, C. S. Williams et al., "Posttraumatic stress symptoms in long-term non-Hodgkin's lymphoma survivors: does time heal?" Journal of Clinical Oncology , vol. 29, no. 34, pp. 4526-4533, 2011.

[21] D. Voliotis and V. Diehl, "Challenges in treating hematologic malignancies," Seminars in Oncology, vol. 29, no. 3, pp. 30-39, 2002.

[22] T. Foster, J. D. Miller, M. E. Boye, and M. W. Russell, "Economic burden of follicular non-Hodgkin's lymphoma," Pharmacoeconomics, vol. 27, no. 8, pp. 657-679, 2009.

[23] S. Orelemans, F. Mols, M. R. Nijziel et al., "The impact of treatment, socio-demographic and clinical characteristics on health-related quality of life among Hodgkins's and nonHodgkin's lymphoma survivors, a systemic review," Annals of Hematology, vol. 90, no. 9, pp. 993-1004, 2011.

[24] A. Leak, D. K. Mayer, and S. Smith, "Quality of life domains among non-Hodgkin lymphoma survivors: an integrative literature review," Leukemia \& Lymphoma, vol. 52, no. 6, pp. 972985, 2011.

[25] E. Deconinck, H. Miadi-Fargier, C. Pen, and P. Brice, "Cost effectiveness of rituximab maintenance therapy in follicular lymphoma: long-term economic evaluation," Pharmacoeconomics, vol. 28, no. 1, pp. 35-46, 2010.

[26] F. Kasteng, M. Erlanson, H. Hagberg, E. Kimby, T. Relander, and J. Lundkvist, "Cost-effectiveness of maintenance rituximab treatment after second line therapy in patients with follicular lymphoma in Sweden," Acta Oncologica, vol. 47, no. 6, pp. 10291036, 2008.

[27] E. J. O. Soini, J. A. Martikainen, and T. Nousiainen, “Treatment of follicular non-Hodgkin's lymphoma with or without rituximab: cost-effectiveness and value of information based on a 5year follow-up," Annals of Oncology, vol. 22, no. 5, pp. 1189-1197, 2011.

[28] N. Martis, C. Gisselbrecht, and N. Mounier, "Quality of life following treatment for B-cell lymphoma," Expert Review of Pharmacoeconomics \& Outcomes Research, vol. 11, no. 5, pp. 523-532, 2011.

[29] N. A. Barber and F. R. Loberiza Jr., "Quality of life after chemotherapy or hematopoietic cell transplant in follicular lymphoma: what's next?" Leukemia \& Lymphoma, vol. 53, no. 3, pp. 352-353, 2012.

[30] D. Tholstrup, P. D. N. Brown, J. Jurlander, P. Bekker Jeppesen, and M. Groenvold, "Quality of life in patients with diffuse large B-cell lymphoma treated with dose-dense chemotherapy is only affected temporarily," Leukemia \& Lymphoma, vol. 52, no. 3, pp. 400-408, 2011.

[31] S. K. Smith, C. S. Williams, C. R. Zimmer, and S. Zimmerman, "An exploratory model of the relationships between cancerrelated trauma outcomes on quality of life in non-Hodgkin lymphoma survivors," Journal of Psychosocial Oncology, vol. 29, no. 1, pp. 19-34, 2011.

[32] K. Yano, T. Kanie, S. Okamoto et al., "Quality of life in adult patients after stem cell transplantation," International Journal of Hematology, vol. 71, no. 3, pp. 283-289, 2000.

[33] A. S. Hamilton, "Quality of life research: adding an important dimension to the evaluation of therapeutic outcomes," Leukemia \& Lymphoma, vol. 52, no. 3, pp. 353-354, 2011.

[34] K. Webster, J. Cashy, D. Cella et al., "Measuring quality of life (QOL) in patients with non-Hodgkin's lymphoma (NHL): the functional assessment of cancer therapy-lymphoma (FACTLym)," Quality of Life Research, vol. 14, p. 1650, 2005.

[35] D. Cella, K. Webster, K. Cashy et al., "Development of a measure of health-related quality of life for non-Hodgkin's lymphoma clinical research: the functional assessment of cancer therapylymphoma (FACT-Lym)," Blood, vol. 106, ASH Annual Meeting Abstracts, no. 11, part 1, 750 pages, 2005.

[36] D. Cella, Ed., Manual of the Functional Assessment of Chronic Illness Therapy (FACIT) Measurement System, Center on Outcomes, Research and Education, Evanston, Ill, USA, 2004.

[37] D. F. Cella, D. S. Tulsky, G. Gray et al., "The functional assessment of cancer therapy scale: development and validation of the general measure," Journal of Clinical Oncology, vol. 11, no. 3, pp. 570-579, 1993.

[38] K. Webster, L. Odom, A. Peterman et al., "The functional assessment of chronic illness therapy (FACIT) measurement system: validation of version 4 of the core questionnaire," Quality of Life Research, vol. 8, no. 7, p. 604, 1999.

[39] D. Cella and C. J. Nowinski, "Measuring quality of life in chronic illness: the functional assessment of chronic illness therapy measurement system," Archives of Physical Medicine and Rehabilitation, vol. 83, no. 12, supplement 2, pp. S10-S17, 2002.

[40] K. Webster, D. Cella, and K. Yost, "The functional assessment of chronic illness therapy (FACIT) measurement system: properties, applications, and interpretation," Health and Quality of Life Outcomes, vol. 1, article 79, 2003.

[41] K. Webster, K. Chivington, C. Shonk et al., "Measuring quality of life (QOL) among patients with leukemia: the functional assessment of cancer-therapy-leukemia (FACT-Leu)," Quality of Life Research, vol. 11, no. 7, p. 678, 2002.

[42] C. G. Zubrod, M. Schneiderman, E. Frei et al., "Appraisal of methods for the study of chemotherapy of cancer in man: comparative therapeutic trial of nitrogen mustard and triethylene thiophosphoramide," Journal of Chronic Diseases, vol. 11, no. 1, pp. 7-33, 1960.

[43] D. F. Cella, P. B. Jacobsen, and E. J. Orav, "A brief POMS measure of distress for cancer patients," Journal of Chronic Diseases, vol. 40, no. 10, pp. 939-942, 1987.

[44] D. P. Crowne and D. Marlowe, "A new scale of social desirability independent of psychopathology," Journal of Consulting Psychology, vol. 24, no. 4, pp. 349-354, 1960.

[45] J. E. Ware, K. K. Snow, and M. Kosinski, SF-36 Health Survey: Manual and Interpretation Guide, QualityMetric, Lincoln, Mass, USA, 2000.

[46] R. Jaeschke, J. Singer, and G. H. Guyatt, "Measurement of health status: ascertaining the minimal clinically important difference," Controlled Clinical Trials, vol. 10, no. 4, pp. 407-415, 1989.

[47] L. J. Cronbach, "Coefficient alpha and the internal structure of tests," Psychometrika, vol. 16, no. 3, pp. 297-334, 1951.

[48] J. C. Nunnally and I. H. Bernstein, Psychometric Theory, McGraw-Hill, New York, NY, USA, 1994.

[49] S. Eremenco, K. Webster, L. Kutikova, L. Bowman, and D. Cella, "Development and multilingual validation of the FACTlymphoma instrument," Quality of Life Research, vol. 13, no. 9, p. 1501, 2004. 


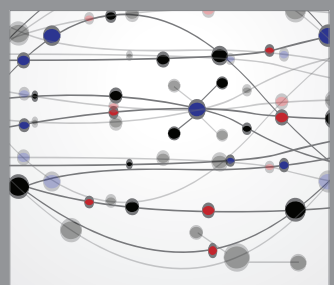

The Scientific World Journal
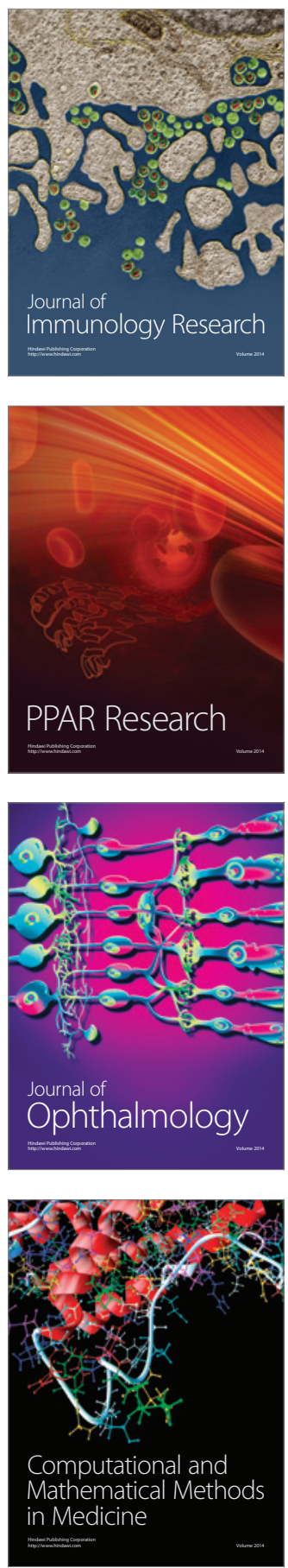

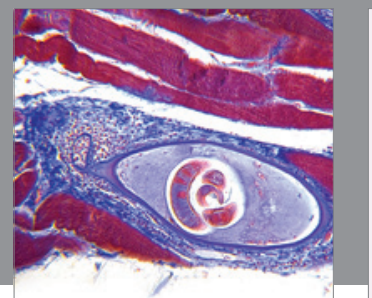

Gastroenterology

Research and Practice
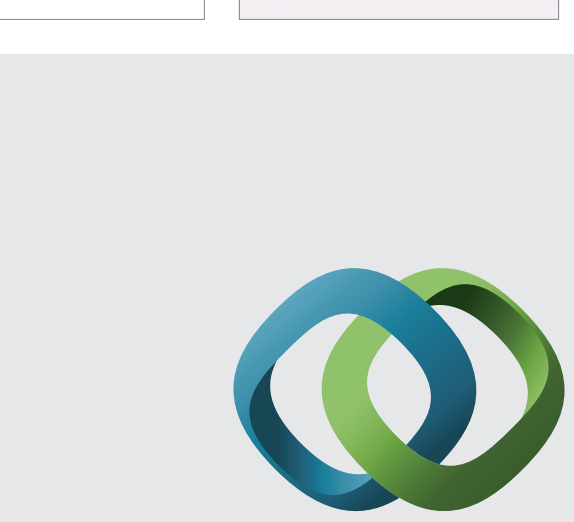

\section{Hindawi}

Submit your manuscripts at

http://www.hindawi.com
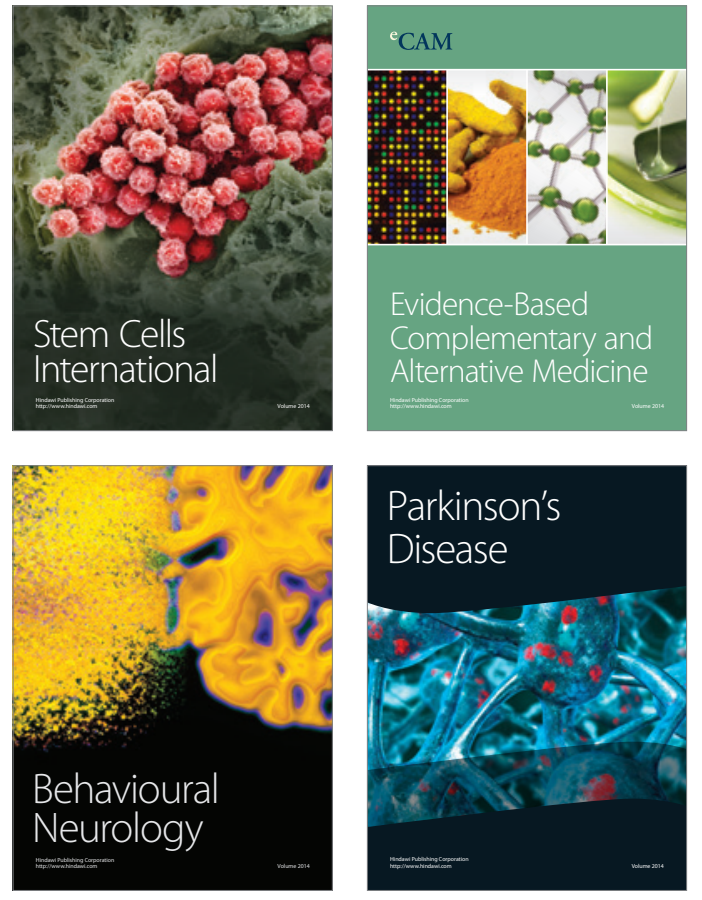
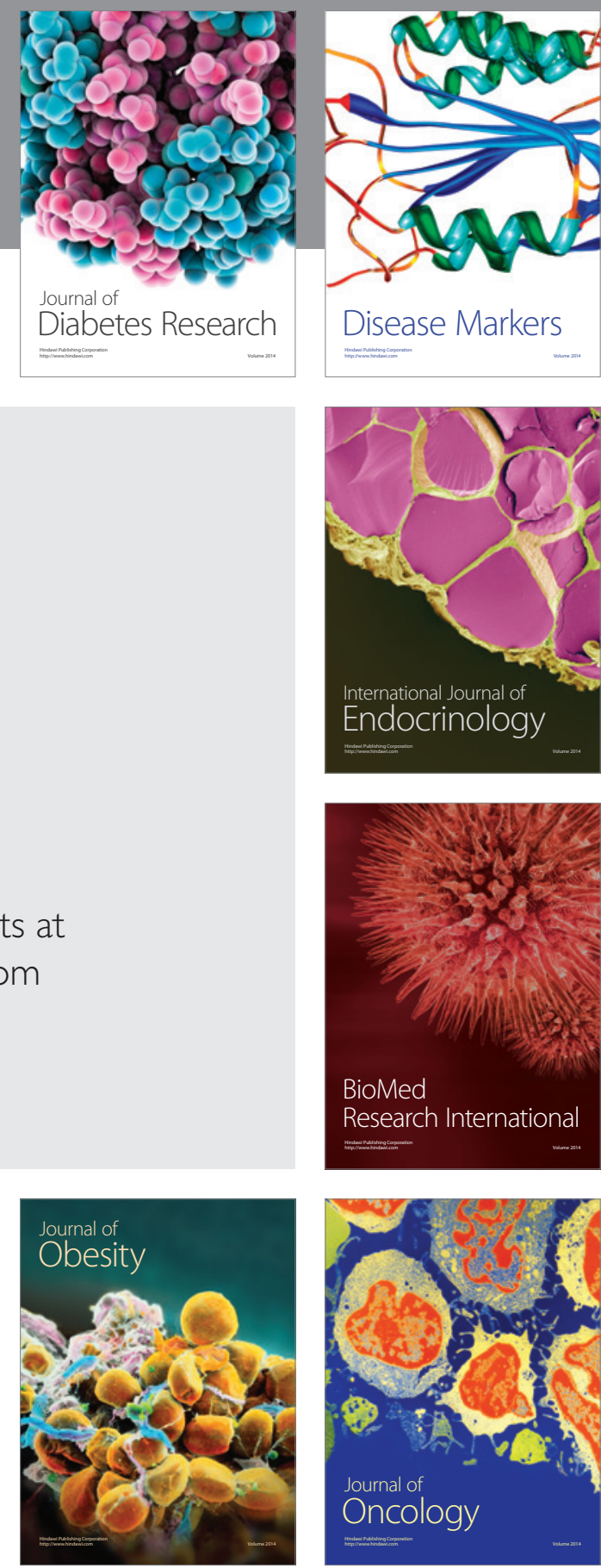

Disease Markers
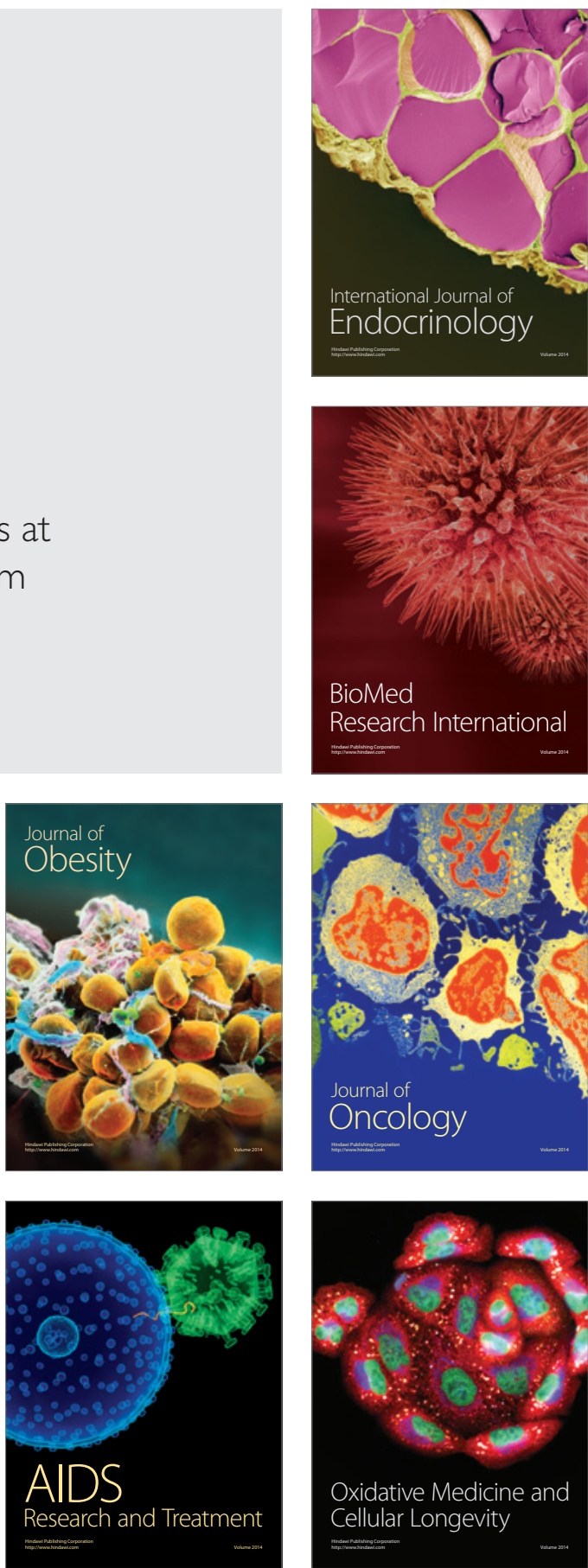